
sociologie des sciences : des débats, un réexamen

\title{
Pourquoi devenir sociologue ? Réflexions et évocations
}

\section{Raymond Boudon}

\section{CpenEdition}

Journals

Édition électronique

URL : http://journals.openedition.org/ress/654

DOI : $10.4000 /$ ress. 654

ISSN : 1663-4446

Éditeur

Librairie Droz

Édition imprimée

Date de publication : 1 juillet 2001

Pagination : 5-30

ISBN : 2-600-00610-9

ISSN : 0048-8046

Référence électronique

Raymond Boudon, "Pourquoi devenir sociologue ? Réflexions et évocations », Revue européenne des sciences sociales [En ligne], XXXIX-120 | 2001, mis en ligne le 11 décembre 2009, consulté le 01 mai 2019. URL : http://journals.openedition.org/ress/654 ; DOI : 10.4000/ress.654 


\section{Raymond BOUDON}

\section{POURQUOI DEVENIR SOCIOLOGUE? RÉFLEXIONS ET ÉVOCATIONS}

La communauté scientifique des sociologues devrait être reconnaissante à H. Mendras pour son petit livre ${ }^{1}$, puisqu'il célèbre l'équipée collective des refondateurs de la sociologie française de l'après-guerre. Il n'est pas le premier. Déjà dans les années 1980, un américain, Ch. Lemert, avait remarqué qu'elle constituait un mouvement d'idées original ${ }^{2}$. Mais alors que Lemert s'en tient à la sociologie française des années 60-70, Mendras remonte à la première vague des pionniers de l'après-guerre, Friedmann, Gurvitch, Aron, Stoetzel. Son parti pris anecdotique n'est pas dépourvu de charme et ses pages sont souvent imprégnées d'humour.

En ce qui me concerne, j'ai été vivement intéressé par la partie du livre portant sur la période dont je n'ai pas été directement témoin. J'ai été fasciné par la scène où $\mathrm{G}$. Friedmann partage le territoire de la sociologie entre ses fils spirituels, leur attribuant un destin que tous acceptent docilement et qu'ils assument pour le restant de leurs jours. Mais j'ai été séduit par la qualité littéraire du texte plutôt que par sa rigueur historique. Parfois, j'ai éprouvé des doutes sur sa véracité psychologique. Les personnages qu'il met en scène ont quelque chose de caricatural. Ils ont des opinions et des états d'âme plutôt que des idées ou des projets et semblent obéir surtout à des soucis de carrière. Le livre contient aussi des naïvetés sociologiques inattendues, comme lorsqu'il affirme que les membres des jurys ne lisent pas les thèses. La vérité est qu'ils risquent d'être mouchés par le candidat, qui en sait toujours plus qu'eux sur son sujet. Aussi la prudence leur conseille-t-elle d'affirmer qu'ils n'ont guère eu de temps à consacrer à la thèse, affirmation que leur intervention démentira généralement. En tout cas, si je me remémore les quelques dizaines de jurys dans lesquels j'ai siégé au cours des cinq dernières années à la Sorbonne et ailleurs, je n'arrive pas à évoquer un seul exemple de collègue qui n'aurait pas lu la thèse examinée.

Le livre contient en outre des erreurs de fait et des à-peu-près qui en font une source peu fiable pour le futur historien. Pour prendre un exemple qui a l'inconvénient d'être personnel mais l'avantage que je puis en juger directement, Mendras affirme: «Stoetzel envoie Boudon chez Lazarsfeld à Columbia». Personne ne m'a envoyé à Columbia et surtout pas Stoetzel que je n'avais pas encore rencontré et qui ignorait alors jusqu'à mon existence.

Mendras H., Comment devenir sociologue? Souvenirs d'un vieux mandarin, Paris, Actes-Sud, 1995.

2 Lemert C., French sociology, rupture and renewal since 1968, New York, Columbia University Press, 1981. 
Outre les erreurs de fait et d'appréciation, l'ouvrage traduit des partis pris curieux. Comment traiter de l'histoire des idées, fût-ce sur le mode de l'anecdote, sans évoquer le contenu des idées? Surtout, s'il tient la promesse de son titre «Comment devenir sociologue?», il esquive une autre question, plus intéressante: «Pourquoi devenir sociologue?». On n'entrevoit pas, l'ayant lu, ce qu'est notre discipline. Pour pratiquer la sociologie, il faut surtout de l'imagination, assure-t-il. Mais il ne nous en donne aucune illustration. Que vise la sociologie? En quoi consiste-t-elle? Aucune allusion, même discrète, n'est faite à une idée ou à une théorie sociologique qui aurait contribué à nous éclairer sur le social. La sociologie joue ici le rôle de la cantatrice chauve.

Si le livre ne répond pas à la question: «Pourquoi devenir sociologue?», c'est qu'il est, telle est du moins mon impression, pétri de scepticisme. Je ne suis pas sûr que Mendras croie à la réalité de sa discipline. C'est peut-être mon reproche essentiel. À quoi sert de nous expliquer comment devenir sociologue, si la sociologie est une discipline insaisissable? Pourquoi nous en conter l'épopée? C'est finalement ce scepticisme qui m'a conduit à accepter l'aimable proposition de réagir à l'ouvrage que m'a adressée la Revue française de science politique. Comme elle m'y a invité, j'ai conçu les notes qui suivent, non comme une discussion du livre de Mendras, mais plutôt comme des variations libres sur les thèmes qu'il éveille dans mon esprit. J'en ai retenu deux, liés entre eux : pourquoi ce scepticisme? Est-il obligatoire d'y souscrire? J'essaierai de les développer à partir de réflexions générales, mais aussi d'évocations relatives à mon propre cas.

\section{LE SCEPTICISME ACTUEL SUR LA SOCIOLOGIE}

Ce scepticisme est d'autant plus intéressant qu'il résulte moins, pour autant que je puisse en juger, d'un trait de personnalité que d'une sensibilité à l'air du temps, avivée sans doute par une désillusion sur l'état présent de notre discipline. Car j'ai connu un temps où Mendras, plein d'enthousiasme et de foi dans l'avenir de la sociologie, était prêt à se lancer dans la création de ces «sociotrons » que Stoetzel appelait de ses vœux. En tout cas, beaucoup d'auteurs témoignent aujourd'hui du même scepticisme. Certains, comme P. Berger, se présentent comme des sceptiques nostalgiques: la sociologie a existé; elle est épuisée. D’autres sont plutôt des sceptiques dogmatiques, tel W. Lepenies: la sociologie n'a jamais produit de savoir. D'autres sont des sceptiques modérés, comme D. Bell: la sociologie n'est pas une science, mais un art «imparfait». D'autres, comme H. White, sont des sceptiques utopistes: la sociologie sera une science demain, une fois qu'elle aura été proprement fondée. D'autres sont des sceptiques désabusés, comme S. Turner et J. Turner: malgré ses efforts, la sociologie américaine n'a, pas plus que les autres, réussi à se donner un programme stable ${ }^{3}$.

Bell D., «Social Science: an Imperfect Art», Revue Tocqueville, XVI, 1, 1995, 3-24. Berger P. L., «Does Sociology Still Make Sense?», Revue suisse de sociologie, XX, 1, 1994, 3-12. Lepenies W., Les trois cultures. Entre science et littérature l'avènement de la sociologie, Paris, Éditions de la MSH, 1990 (1985). Turner S. P., Turner J. H, The impossible science. An institutional analysis of American sociology, London, Sage, 1990. White H. C., Identity and Control, A Structural Theory of Social Action, Princeton, Princeton U. Press, 1993. 
Ce scepticisme ne se dégage-t-il pas en fin de compte de l'état même de la sociologie? Dans son pastiche un peu convenu de La Bruyère, Mendras crayonne des portraits de sociologues qui, bien qu'anecdotiques, témoignent de l'hétérogénéité de la discipline. Surtout, les pionniers de la première génération, qu'il campe joliment, furent des personnalités contrastées, qui entretinrent des conceptions des sciences sociales inconciliables entre elles et pour cette raison sans doute ne s'entendirent guère. Quoi de commun en effet entre les vues que s'en firent Gurvitch, Friedmann, Aron ou Stoetzel?

\section{La sociologie: une discipline polymorphe}

Cette hétérogénéité n'est pas propre à la sociologie française d'après-guerre. On la retrouve à l'état chronique dans toute l'histoire des sciences sociales, comme le démontre, nolens volens, R. Aron dans Les étapes de la pensée sociologique $^{4}$. Le titre de ce livre, qui décalque celui de L. Brunschvicg, Les étapes de la pensée mathématique, suggère que la sociologie n'a pas été un voyageur sans boussole. En fait, le lecteur n'y discerne ni le chemin qu'elle aurait emprunté, ni les étapes auxquelles elle aurait fait halte, ni à vrai dire la pensée sociologique, mais il est guidé de main de maître dans une galerie de portraits -ceux de Montesquieu, d'Auguste Comte, de Marx, de Tocqueville, de Max Weber, de Durkheim et de Pareto-, dont il a quelque peine à percevoir les ressemblances. L'histoire de la sociologie française de l'après-guerre ne fait donc peut-être qu'illustrer un état de choses permanent.

\section{Les traditions nationales}

Comme s'il s'agissait d'alimenter ce scepticisme, la sociologie a été longtemps marquée par de fortes traditions nationales. L'Allemagne et la France sont pour elle deux terres d'élection. Or la tradition française est et restait naguère fort différente de la tradition allemande. Il y a, comme le dit justement Aron, une continuité de Comte à Durkheim, de Durkheim à Mauss et de Mauss à LéviStrauss; il y a bien des points communs entre Marx, Weber, Sombart et Simmel; mais, suggère-t-il, un gouffre sépare les deux traditions.

On ne peut naturellement, à la manière des «holistes », imputer ces différences à on ne sait quelles âmes nationales. La persistance des traditions doit s'expliquer plutôt dans le cadre de l'«individualisme méthodologique», car elle provient principalement de ce que les individus mobilisent le passé pour juger du présent et définir l'avenir. Les paysans serbes exposent couramment des photos de leurs parents et de leurs grands parents en uniforme militaire: elles inspirent leurs appréciations politiques sur la situation actuelle, celle de 1995, en Bosnie. Les conditions édictées par les Américains à l'encontre des Allemands et des Japonais à la fin de la seconde Guerre mondiale s'expliquent par leur souci de ne pas renouveler les erreurs commises par Wilson à la fin de la première ${ }^{5}$. La permanence des

4 Je résume ici mon article «Le non-dit des Étapes de la pensée sociologique de R. Aron», Commentaire, 8, 28-29, 1985, 222-225.

5 May E., Lessons of the past; the use and misuse of history in American foreign policy, New York, Oxford U. Press, 1973. 
traditions et l'inertie des structures sont bien véhiculées par des représentations et des desseins individuels.

C'est le cas ici, s'agissant des traditions sociologiques. Littré s'était attribué le rôle de légataire intellectuel d'Auguste Comte, mais il voulait amender les projets de son idole afin de mieux la servir et nourrissait une ambition bien précise: rendre la sociologie française aussi scientifique que celle de Spencer ${ }^{6}$. Pour cela, elle devait selon lui s'appuyer, à l'instar du maître anglais, sur des faits méthodiquement collectés, mais aussi éviter à tout prix l'individualisme de Spencer, lequel paraissait bien sûr inacceptable à un disciple de Comte. On sait le rôle crucial joué par Littré dans la fondation de la Troisième République et l'aura dont il bénéficia auprès des milieux intellectuels, mondains et politiques ${ }^{7}$. C'est Durkheim qui devait en fait réaliser le rêve de Littré. Après la deuxième Guerre mondiale, et bien qu'il ait été réimporté des États-Unis, l'auteur des Règles de la méthode sociologique prit la stature d'un personnage emblématique de la sociologie française. Sans toujours se l'avouer, les sociologues français se situent par rapport à lui un peu comme les politiques par rapport à de Gaulle ${ }^{8}$.

La sociologie allemande classique se cristallise dans un contexte très différent, celui de la consolidation de l'histoire scientifique et du développement des sciences humaines d'érudition, celui aussi de la critique kantienne. Weber a les mêmes ambitions scientifiques que Durkheim. Mais alors que Durkheim se donne une notion positiviste de la science, qu'à travers Littré il avait héritée de Comte, Weber en développe une tout autre. Comme les historiens à la Ranke, les sociologues allemands classiques partent du principe que les phénomènes sociaux résultent de l'agrégation de croyances, d'attitudes et d'actions individuelles. En insistant sur la notion de «compréhension», ils précisent que les causes de ces croyances, attitudes et actions individuelles ne sont pas autre chose que le sens qu'elles ont pour l'acteur. Les expliquer, c'est donc reconstruire ledit sens. C'est en d'autres termes, ajouterai-je en systématisant Weber, reconstruire les raisons qu'a l'acteur de les endosser.

La sociologie française classique se constitue finalement autour d'un programme positiviste dur ${ }^{9}$. Elle attribue une importance décisive au principe selon lequel le vrai savant doit ignorer l'invisible, en l'occurrence le psychique, et s'en tenir à l'étude des relations entre des données observables. D'où le mandement de Durkheim: «expliquer les faits sociaux par les faits sociaux». La sociologie allemande classique a la même ambition de scientificité, mais, tenant la «compréhension» pour essentielle, elle n'accepte pas l'idée que les faits sociaux ne s'expliquent que par les faits sociaux. Comme elle refuse de rechercher les causes des

6 Yamashita, M., «La sociologie française entre Auguste Comte et Émile Durkheim: Émile Littré et ses collaborateurs », L'année sociologique, 45, 1, 1995, 83-115.

Nicolet C., L'idée républicaine en France (1789-1924), Paris, Gallimard, 1982.

8 Je n'échappe pas à cette règle. Cf. «Should one still read Durkheim's Rules after one hundred years? Raymond Boudon interviewed by Massimo Borlandi», Revue suisse de sociologie, Vol. 21, n 3, nov. 1995, 559-573 (reproduit in Boudon, R., Etudes sur les sociologues classiques, Paris, PUF, 1998).

9 Pour plus de précisions sur cette notion, voir ma communication au Colloque « Durkheim», Bordeaux, 18-20 mai 1995: «Peut-on être positiviste aujourd'hui?», reprise in: Cuin Ch. (éd.), Durkheim d' un siècle à l'autre, Paris, PUF, 1997, 265-287. 
phénomènes sociaux ailleurs que dans les actions des hommes, elle rejette également l'idée que la scientificité implique une conception déterministe du monde.

Mais il ne faut pas surestimer la cohérence des traditions nationales. Si Durkheim doit beaucoup à Comte, Tocqueville ne lui doit rien et il utilise instinctivement la méthodologie que Weber défendra explicitement après lui. Quant à Le Play, il n'a pas grand'chose à voir avec Durkheim. Le jeu des continuités et des discontinuités qui rythme l'histoire de la sociologie rappelle en fin de compte davantage l'histoire de la musique que celle de la physique ${ }^{10}$.

\section{Absence d'identité mais non de réalité}

De tout cela, il résulte que la sociologie n'a guère d'identité, non qu'elle n'ait pas d'existence. Mais le substantialisme est un cadre de pensée si tyrannique qu'on a tendance à supposer que l'existence implique l'identité. À partir du moment où l'on prononce le mot sociologie au singulier, on a l'impression qu'il doit y avoir quelque chose de commun entre tous ses produits. Wittgenstein nous a appris cependant que la notion de jeu n'est pas vide de sens, bien qu'il n'y ait rien de commun entre les jeux concrets qui matérialisent cette notion abstraite. En tout cas, quand nous avons fondé, F. Bourricaud et moi-même, une collection de sociologie aux Presses Universitaires de France, nous l'avons spontanément intitulée « Sociologies », ce pluriel n'impliquant en aucune façon que toutes les sociologies se valent.

Ainsi, la sociologie au singulier n'existe pas. Il existe des traditions sociologiques, et des types de sociologie. Lesquels? Quels sont les plus importants? Pourquoi verse-t-on dans l'un plutôt que dans l'autre? Je dirai dans la suite celui que je considère le plus important et pourquoi j'y ai versé, mais auparavant, il me faut revenir sur un point laissé en suspens.

\section{Pourquoi ce scepticisme aujourd'hui?}

Pourquoi ces problèmes d'identité conduisent-ils au scepticisme aujourd' hui? Beaucoup d'éléments plaident en faveur de cette attitude, mais le principal, du point de vue qui m'occupe ici, est sans doute le succès d'un mouvement de pensée qui s'est développé dans les trente dernières années, à partir de la France et des États-Unis surtout. Bien que stérile, il est très influent socialement. Parfois qualifié de postmoderniste, il comporte de multiples variantes. Mais sa thèse principale peut être facilement énoncée: elle consiste à traiter l'objectivité comme une illusion et, par suite, à faire fi des règles élémentaires de la démarche scientifique. Les auteurs postmodernistes s'appuient sur une célèbre formule de Nietzsche, «il n'y a pas de faits, mais seulement des interprétations ». La prenant naïvement au pied de la lettre, ils en tirent l'idée que l'analyse d'un texte, d'un phénomène ou d'une institution ne saurait prétendre à une illusoire justesse. Elle doit plutôt s'efforcer d'être nouvelle, de plaire et de toucher ou, mieux encore, de «déranger».

10 Boudon R., «European sociology: the identity lost?», in Nedelmann B. et Sztompka P., (éds), Sociology in Europe. In search of identity, New York/Berlin, de Gruyter, 1993, 27-44. 
Ce nihilisme nous a valu par exemple les célèbres variations de M. Foucault sur le thème du chaperon rouge ${ }^{11}$. L'adoucissement séculaire des peines répressives résulte, explique-t-il, de ce que la Société a appris à dissimuler le pouvoir qu'elle exerce sur l'individu, afin de mieux l'écraser. Ainsi, selon feu le professeur au Collège de France, l'allégement des peines serait la preuve décisive de l'alourdissement de la répression. Autre exemple: ayant planté sa tente sur le territoire de la sociologie des sciences, le postmodernisme nous a enseigné que le progrès scientifique, y compris dans les sciences de la nature, est une illusion collective, qu'il n'y a pas de moyen de trancher de façon objective entre des théories concurrentes, et que, en science comme partout, c'est la raison du plus fort qui est toujours la meilleure ${ }^{12}$. Dans un registre plus grave, une école américaine ayant pignon sur rue, se qualifiant elle-même de révisionniste, tente, au nom de la même liberté d'interprétation, de présenter le stalinisme comme un régime bien intentionné, qui aurait éliminé les gens dans le noble souci d'augmenter la mobilité sociale $^{13}$. On pourrait sans grande difficulté aligner bien d'autres manifestations du gai savoir produit par les postmodernistes ${ }^{14}$.

Certains établissements d'enseignement supérieur, parce qu'ils sont moins médiatisés, paraissent mieux armés que d'autres pour résister à ce nihilisme. La crise est d'autre part inégalement profonde selon les disciplines. Mais, comme on l'a opportunément souligné ${ }^{15}$, c'est la première fois dans l'histoire des sociétés occidentales qu'on voit s'installer dans des organismes voués à la création et à la transmission du savoir un mouvement de pensée qui tente de le disqualifier. Il est vrai que, si le nihilisme a toujours existé, le nihilisme de la chaire, lui, est bien un phénomène nouveau.

Ce syndrome est-il passager? Il est difficile de le dire. Certains y voient un phénomène conjoncturel: l'avant-garde postmoderniste ne serait que l'arrière-garde de $68^{16}$. Sans doute. Mais cette dévalorisation du savoir a fini par démonétiser l'esprit critique, et par détrôner la discussion, qui vise le vrai, au profit de la communication, dont toute l'ambition se borne à «faire passer le message » sans préjuger de sa vérité. Mendras décrit fort bien les perversions engendrées par ce nihilisme: échanges intellectuels truqués, dissimulant des règlements de compte ou des renvois d'ascenseur; constitution de clubs d'admiration mutuelle; dignités «scientifiques » conférées sur la foi de tocades médiatiques; artistes de la mise en scène du moi se faisant passer pour des penseurs; surtout, ajouterai-je, affaissement de la solidité et de la crédibilité des sciences humaines, et pis encore, tous ces étudiants qui pour leur malheur perdent de précieuses années à découvrir (quand ils y parviennent) que le roi est nu.

\footnotetext{
Foucault M., Surveiller et punir ; naissance de la prison, Paris, Gallimard, 1975.
}

12 Boudon R. et Clavelin M. (éds.), Le relativisme est-il résistible? Regards sur la sociologie des sciences, Paris, PUF, 1994.

13 «Revisionismus revidiert», Frankfurter Allgemeine Zeitung, 17 août 1994.

14 J'en propose d'autres in «Les sciences humaines: troisième culture?», Discours prononcé lors de la rentrée solennelle des cinq académies de l'Institut de France, 18 octobre 1994, Paris, Institut de France, $1994, \mathrm{~N}^{\circ} 15$.

15 Searle J. R., «Rationality and Realism. What is at Stake?», Daedalus, Fall 1993, 55-83.

16 Comme l'indique un très fiable Who is who du nihilisme français contemporain: Ferry L. et Renaut A., La pensée 68, Paris, Gallimard, 1985. 


\section{LES SUCCÈS DE LA CONCEPTION SCIENTIFIQUE DES SCIENCES SOCIALES}

Le scepticisme que Mendras manifeste involontairement à propos de la sociologie est sans doute l'émanation de cet état d'esprit général. Mais une opinion, même majoritaire, peut être erronée. C'est le cas ici. Le scepticisme en question se heurte en effet à l'objection du réel. Je veux dire qu'on identifie facilement dans la réalité des sciences sociales des contributions au savoir ayant la solidité de celles des sciences de la nature. Bref, il existe dans le magma des sciences sociales une coulée scientifique, orientée vers la production d'un authentique savoir.

\section{Théories (expliquer des énigmes)}

Pour les plus grands, pour Weber, Durkheim et Tocqueville notamment, la fonction essentielle de la sociologie réside bien dans la création de savoir. Ils ne partagent pas la même conception de la science, mais ils ont en commun de nourrir pour la sociologie une ambition scientifique.

Sans vouloir rentrer trop avant dans une discussion complexe: pourquoi la théorie du pendule de Huygens est-elle bonne? Parce qu'elle satisfait à deux principes: le principe (popperien) de l'accord de la théorie avec tous les faits relevant de son domaine de compétence et le principe (non popperien) selon lequel les propositions non empiriques d'une théorie (il y en a toujours) doivent être acceptables. Lorsque Huygens introduit la proposition que l'une des forces agissant sur le pendule l'attire vers le centre de la terre, il introduit évidemment une proposition non empirique. Personne n'a jamais vu une force et encore moins une force qui attire les objets vers le centre de la terre. Néanmoins la proposition est généralement considérée comme acceptable. Pourquoi une théorie est-elle mauvaise? Parce qu'elle viole l'un au moins de ces principes.

La notion de scientificité n'a pas lieu d'être définie différemment s'agissant des sciences de l'homme et de la sociologie en particulier et l'on y repère facilement des théories aussi solides que celle de Huygens. Pour établir ce point, je me contenterai d'évoquer une théorie classique, l'explication par Tocqueville et Weber de la religiosité américaine ${ }^{17}$.

Ce phénomène a été considéré par eux comme profondément énigmatique, car il représente un défi à la loi évolutive énoncée par Comte, par Tocqueville lui-même, par Durkheim, par Weber, selon laquelle la modernité entraînerait ce que Schiller avait appelé le «désenchantement du monde». Cette loi est fondée. Mais elle est grossière et comporte des exceptions, dont l'exception américaine, d'autant plus énigmatique que le désenchantement aurait dû être plus prononcé aux États-Unis qu'ailleurs. Pourquoi faut-il en effet que ce soit la société la plus moderne, la plus matérialiste aussi, qui demeure la plus religieuse des nations occidentales? L'énigme est suffisamment déconcertante pour avoir été explorée par maints auteurs classiques et modernes. Mais ces derniers n'ont pas apporté d'éléments fondamentalement nouveaux par rapport à Adam Smith, Tocqueville et Weber: un peu comme la théorie du pendule est close depuis Huygens, on a

17 Je résume ici mon argumentation in «Relativiser le relativisme», Revue Tocqueville, XV, 2, 1994, 109-31. 
l'impression que la théorie polycéphale de Smith-Tocqueville-Weber épuise largement le sujet de l'exception religieuse américaine. Je laisserai Adam Smith de côté pour des raisons de place.

Le caractère explosé des institutions religieuses américaines (une multitude de sectes, pas d'église dominante) a rendu peu plausible, nous dit Tocqueville, la concurrence entre le spirituel et le politique qui apparaît en France pendant la Révolution de 1789. Par voie de conséquence, les sectes américaines ont conservé des fonctions sociales essentielles (santé, éducation, assistance) qui sont passées aux mains de l'État dans les nations européennes. La limitation des compétences de l'État américain, due à son caractère fédéral, a joué dans le même sens. Il en résulte que le citoyen américain côtoie les institutions religieuses dans sa vie de tous les jours. Comment, dans ces conditions, développer des sentiments négatifs à leur endroit? De surcroît, la multiplicité des sectes a fait qu'une grande tolérance s'est développée à l'égard des «croyances dogmatiques ». Comme ces croyances varient d'une secte à l'autre, on en est progressivement arrivé à l'idée que les vérités dogmatiques relèvent dans une très large mesure de l'appréciation personnelle. Cette liberté d'interprétation est latente dans le protestantisme. Elle est renforcée dans une situation où les sectes sont multiples. Cette donnée a engendré à son tour un effet crucial: les croyances dogmatiques étant très diverses, le fond commun du protestantisme américain a pris avec le temps un caractère surtout moral. Quand les articles du dogme sont rentrés en concurrence avec l'interprétation scientifique du monde, la religiosité américaine en a donc beaucoup moins souffert que la religiosité française.

Weber ajoute à cette théorie un point essentiel. L'importance du mythe égalitaire aux États-Unis (qui a lui même été rendu possible par le fait que le pays soit un pays d'immigration) fait que les symboles linguistiques, vestimentaires et autres de la stratification sociale y sont beaucoup moins marqués qu'en France ou en Allemagne. Mais comme il existe bien sûr une stratification aux États-Unis et que la vie sociale dans ses aspects les plus courants implique qu'on sache «à qui on a affaire », c'est-à-dire qu'on puisse apprécier d'un coup d'œil la place de l'autre dans l'échelle sociale, il est important de pouvoir disposer de symboles de statut. Les distinctions vestimentaires, linguistiques, gestuelles, les signes officiels de distinction (comme les décorations et les titres) n'ayant pas aux ÉtatsUnis l'importance qu'elles ont en Allemagne ou en France, ce sont les affiliations religieuses qui vont en tenir lieu. Pourquoi sont-elles habilitées à assumer cette fonction? Parce que, en raison de l'histoire des phénomènes migratoires, il existe une corrélation entre l'affiliation religieuse et la position dans le système de stratification, un groupe ayant d'autant plus de chances d'être représenté dans les élites qu'il correspond à une émigration plus ancienne.

Si la théorie de Smith-Tocqueville-Weber de l'exception religieuse américaine n'a pas été remise en cause par la suite et n'a guère été complétée que sur des détails, c'est que, à l'instar de la théorie de Huygens, elle fournit une explication satisfaisante du phénomène sous examen. En effet, ses propositions empiriques sont congruentes avec le réel (il est exact qu'il existe des sectes aux États-Unis, et une religion dominante en France, en Allemagne ou en Angleterre; que les fonctions d'éducation ou de solidarité sont plus souvent assumées par des institutions religieuses aux États-Unis qu'en France ou en Allemagne; il est vrai que l'athéisme est plus répandu en Angleterre qu'aux États-Unis, etc.). Bref, la théo- 
rie polycéphale de l'exception religieuse américaine contient une foule de propositions dont on peut évaluer la congruence avec le réel et qui de ce point de vue (popperien) sont parfaitement acceptables. D'un autre côté, elle comporte maintes propositions qu'on peut difficilement confronter à l'observation: lorsqu'on a le sentiment d'appartenir à une communauté et que les opinions de ses membres divergent sur le thème $\mathrm{A}$ et convergent sur $\mathrm{B}$, on a tendance à définir la communauté à partir de $\mathrm{B}$ (Tocqueville); la compétition cristallise l'hostilité (Tocqueville); l'interaction élémentaire ne peut se dispenser de symboles de stratification (Weber), etc. Toutes ces propositions sont aussi peu testables directement que l'existence des forces qui meuvent le pendule. Mais elles sont acceptables, car on peut les déduire de théories fortes et en trouver mille applications à d'autres cas. Ces énoncés, qu'ils soient psychologiques ou sociologiques, sont donc aussi facilement recevables que les énoncés physiques de Huygens.

Bref, la théorie de Smith-Tocqueville-Weber est aussi bonne que celle de Huygens: elle passe haut la main les critères popperiens et non popperiens qui définissent la notion de théorie scientifique. Le fait que cette théorie continue d'inspirer un flux de recherches complémentaires témoigne d'ailleurs de son importance ${ }^{18}$.

S'agissant de la sociologie moderne, les travaux se proposant d'expliquer des phénomènes de caractère énigmatique abondent et l'on n'a ici que l'embarras du choix: pourquoi les taux de criminalité augmentent-ils depuis plusieurs décennies? Pourquoi l'évolution des courbes de criminalité est-elle semblable dans beaucoup de pays, à l'exception de la Suisse et du Japon, se demande Cusson? Pourquoi les entreprises japonaises obéissent-elles à des principes différents des entreprises américaines ou européennes, se demande B. Coriat? D'où vient que le capitalisme moderne se soit développé en Europe, se demande J. Baechler, complétant par une réponse originale à cette question classique celles qui avaient déjà été données? Pourquoi les croyances irrationnelles sont-elles plus répandues dans les régions les plus modernes de l'Europe du XVIe siècle et non dans les plus traditionnelles? Pourquoi sont-elles plus répandues au XVIIe siècle qu'au XVe, se demandent K.Thomas, puis A. Oberschall? Pourquoi, au XVIIe siècle, les peintres hollandais s'entichent-ils de la nature morte? Comment expliquer l'apparition de l'art abstrait, se demande A. Besançon? Pourquoi la politique économique est-elle plutôt favorable aux consommateurs dans la France du XVIIIe siècle et aux producteurs dans l'Angleterre de la même époque; pourquoi accepte-t-on ainsi en France une politique qui décourage la production, se demande H.L. Root? Pourquoi en Australie la bière est-elle une boisson prestigieuse et le whisky une boisson populaire, se demande Lipset? Pourquoi la Bolivie détient-elle le record des coups d'état en Amérique latine, se demande J.-P. Lavaud? Quelles sont les raisons d'être des bandes de jeunes, se demande W. F. White? Comment expliquer, se demande Downs, que le public évalue généralement les mesures politiques qu'on lui propose à partir, non de leurs conséquences, mais des principes qui les fondent, un problème que Hume avait présenté

18 Chaves, M., Cann, D., «Regulation, pluralism and religious market structure: explaining religious vitality », Rationality and society, 4, 1992, 272-290. 
comme le plus ardu auquel les sciences humaines soient confrontées? ${ }^{19}$ Toutes les théories auxquelles je fais allusion ici satisfont aux critères popperiens et non popperiens qui définissent une théorie scientifique.

J'ai proposé moi-même sur diverses questions des théories dont je crois qu'elles répondent également à ces critères: pourquoi la culture scientifique n'a-t-elle pas évincé l'irrationnel? Pourquoi le relativisme est-il aujourd'hui dominant? Quelles sont les causes de la révolte étudiante de $68 ?^{20} \mathrm{C}$ 'est la même conception qui m'a inspiré dans L'inégalité des chances ${ }^{21}$. Le point de départ de la recherche est constitué par une série d'énigmes. Pourquoi l'inégalité des chances scolaires est-elle si élevée, et apparaît-elle comme si résistante aux traitements politiques? Le problème ici n'était pas d'identifier les causes de cette forme d'inégalité: sociologues, psychologues sociaux, démographes et économistes en avaient découvert de nombreuses. Il était plutôt de déterminer leur importance relative, ce qui n'avait pas été tenté. Autre phénomène étrange: l'atténuation de l'inégalité des chances scolaires paraît avoir peu d'effet sur la mobilité sociale, bien que l'origine sociale ait une influence sur le niveau scolaire et ce dernier une influence sur le statut social. J'ai tenté de résoudre ces énigmes par un modèle incorporant entre autres des hypothèses inspirées de la théorie des groupes de référence: toutes choses égales d'ailleurs, la valeur qu'un sujet (ou sa famille) attribue à un niveau scolaire plus élevé que celui qu'il a d'ores et déjà atteint est estimée par rapport à sa position sociale. Ce modèle permet de rendre compte d'une multitude de données statistiques, non dans leur détail numérique, mais dans leur allure structurelle.

Mendras s'interroge sur l'échec de ce livre. En dépit de ses multiples éditions, de ses traductions dans les langues majeures et des nombreuses recherches qu'il a inspirées jusqu'à aujourd'hui, il a été effectivement un relatif échec (de librairie). Aron, qui avait dit publiquement l'estime dans laquelle il le tenait ${ }^{22}, \mathrm{~m}^{\prime}$ en avait prévenu: «un livre qui doit être lu crayon à la main ne saurait être populaire». Cela me paraissait l'évidence même. Cette théorie a de surcroît rencontré l'hosti-

19 Le lecteur conclura facilement de cette liste hétéroclite que la sociologie n'est pas cantonnée pour moi aux frontières bureaucratico-corporatistes que lui assignent ses vestales. Cusson M., Croissance et décroissance du crime, Paris, PUF, 1990. Coriat, B., Penser à l' envers, Paris, Bourgois, 1991. Baechler J., Les origines du capitalisme, Paris, Gallimard, 1971. Thomas K., Religion and the decline of magic, Harmondsworth, Penguin, 1973. Oberschall A., «Cultural change and social movements », communication au congrès de l'association américaine de sociologie, 1989. Besançon A., L'Image interdite, une histoire intellectuelle de l'iconoclasme, Paris, Fayard, 1994. Root H. L., La construction de l'état moderne en Europe. La France et l'Angleterre, Paris, PUF., 1994. Lipset S. M., The first new nation, Doubleday, Garden City NY, 1967 (1963). Lavaud J. P., L'instabilité politique de l'Amérique latine: le cas de la Bolivie, Paris, L'Harmattan, 1991. White W.F., Street corner society; the social structure of an Italian slum, Chicago U. Press, 1955. Downs A., An economic theory of democracy, New York, Harper, 1957.

20 «Les deux sociologies de la connaissance scientifique», in: Boudon et Clavelin, op. cit., 17-43; «Subjective rationality and the theory of ideology », in: Haferkamp H. (éd.), Social Structure and Culture, Berlin/New York, de Gruyter, 1989, 269-288; «La crise universitaire française: essai de diagnostic», Annales, XXIV, 3, 1969, 738-764; «Sources of student protest in France», The Annals of the American Academy of Political and Social Science, vol. 395, May 1971, 139-149.

21 L'inégalité des chances, Paris, Colin/ Hachette, Coll. Pluriel, 1985 (1973).

22 À l'occasion de l'attribution à ce livre du prix Émile Girardeau par l'Académie des sciences morales et politiques. 
lité, en France d'une coterie, celle des sociologues structuralo-marxistes (que j'ai, il est vrai, un peu malmenés à plusieurs reprises ${ }^{23}$ ), et, aux États-Unis d'un groupe d'intérêt, celui de ces «statistical zealots» dont Lazarsfeld se moquait volontiers $^{24}$. Je menaçais en effet leur fonds de commerce: pour eux, une étude sociologique est bonne si elle applique la dernière technique statistique à la mode et mauvaise dans le cas contraire. (La différence entre les coteries et les groupes d'intérêt est que les premières tendent à survivre à la mort des idées qui ont rassemblé leurs membres). En revanche, beaucoup comprirent que, malgré ses faiblesses, le livre était méthodologiquement novateur et qu'il comportait des applications impor$\operatorname{tantes}^{25}$. En proposant une théorie qui expliquait des données macroscopiques en les déduisant d'hypothèses microscopiques, il ouvrait - par l'exemple - la voie d'une réconciliation entre l'analyse statistique et la tradition de la sociologie compréhensive $^{26}$. D'un point de vue pratique, il permettait de mieux comprendre l'échec de certaines politiques d'éducation, d'en suggérer de moins contreproductives, et aussi, de nuancer le fatalisme sommaire des théoriciens de la prédestination sociale s $^{27}$.

Le premier apport tangible de la sociologie à visée scientifique consiste donc à expliquer des énigmes à l'aide de théories recevables selon les critères habituellement utilisés pour juger d'une théorie scientifique. Les exemples de théories que je viens d'évoquer se caractérisent par le fait qu'elles expliquent des phénomènes bien circonscrits. On peut appeler ces produits «théories» ou préciser «théories de type $\mathrm{A} »$.

\section{Modèles (expliquer des phénomènes hétéroclites)}

La coulée scientifique que j'évoquais comporte, si on la remonte, un deuxième type de produit. Il s'agit ici de théories qui, sans être générales, permettent d'expliquer efficacement des ensembles de faits à première vue hétéroclites. Appelons-les théories de type B. On pourrait aussi les qualifier de modèles, car c'est là un des sens fréquents de cette notion.

23 «The three basic paradigms of macrosociology: functionalism, neomarxism and action analysis », Theory and Decision, 6, 1976, 381-406; «The Freudian Marxian structuralist (FMS) movement in France: variations on a theme by Sherry Turckle», Revue Tocqueville, hiver 1980, 5-24.

24 Il emploie notamment cette expression dans une lettre qu'il m'a adressée à l'époque de la parution de l'Inégalité des chances aux États-Unis.

25 Voir notamment le symposium publié par la revue Information sur les sciences sociales, 1974-1975, et comprenant des contributions de Rokkan S. (Bergen), Elster J. (Oslo), Alker H. (MIT), Andorka R. (Budapest), Rogoff N. (Oslo), Miller S.M. (Boston), Fararo T. (Pittsburg), Kosaka K. (Tokyo).

26 J'avais déjà manifesté l'importance que j'attachais à cette question in «Les mécanismes sociaux des abandons de poursuite» (avec A. Davidovitch), L'Année sociologique, 1964, 111-244 et dans «La statistique psychologique de Tarde», Annales internationales de criminologie, 1964, 1-16. Voir aussi «Generating models as a research strategy», in Merton R. K., Coleman J. S., Rossi P. H. (eds), Qualitative and quantitative social Research, New York, The Free Press, 1979, 51-64.

27 Les recherches ultérieures confirment très généralement les conclusions du livre. Voir ma conférence à la Fondation Saint-Simon: «Les causes de l'inégalité des chances scolaires », Commentaire, $\mathrm{n}^{\circ}$ 51, automne 1990, 533-542. 
Je pense ici à des travaux désormais classiques comme ceux d'Olson ${ }^{28}$. Leur point de départ est encore une énigme: comment expliquer l'institution du closed shop. Qu'il faille forcer les gens à contribuer à la production d'un bien collectif qu'ils apprécient est en effet paradoxal. Mais cette question a donné naissance à un modèle qui permet d'expliquer des phénomènes fort différents les uns des autres, et qu'on explique difficilement sans lui, comme le fait qu'un petit groupe - le lobby des fabricants d'armes aux États-Unis par exemple - puisse prendre un grand groupe non organisé en otage, comme la préférence à première vue irrationnelle du public pour l'impôt indirect («indolore» selon une formule juste et consacrée), comme la loi d'airain de l'oligarchie de Michels, ou comme le rôle joué par les intellectuels dans certains types de mouvements sociaux. On peut évoquer aussi dans cette catégorie les travaux de T. Kuran ${ }^{29}$, qui montrent que l'expression publique des préférences privées peut en fournir une représentation très déformée, avec les conséquences politiques qu'on imagine. Ainsi, comme il est facile et socialement rémunérateur d'exprimer une position fondée sur de bons sentiments, seules les belles âmes ont tendance à faire entendre leur voix. C'est pourquoi un gouvernement peut facilement avoir l'impression qu'une politique fait l'objet d'une demande forte de la part de l'opinion, alors même qu'elle n'est approuvée que par une minorité. On explique de cette manière le développement aux États-Unis d'une affirmative action aux effets parfois regrettables. Lorsqu'on me demande si je pense que telle décision politique est bonne, ma réponse dépend de trois considérations: de mon opinion sur la question, de la valeur que j'attache au fait de l'exprimer, et des risques (ou des avantages) que j'encours en l'exprimant. Ces idées simples permettent d'élucider des phénomènes variés. Ainsi, avant l'élection nicaraguayenne de 1990, la plupart des sondages prévoient le succès des sandinistes, alors que le candidat de l'opposition, Violetta Chamorro, devait emporter une victoire éclatante, avec une avance de 14 points. Pourquoi cette grossière erreur de prédiction? Parce que de nombreux sondés perçurent les sondeurs comme proches des sandinistes et, ne l'étant pas eux-mêmes, dissimulèrent leurs préférences; de plus, de grands journaux américains, parce qu'ils inclinaient vers les sandinistes, ne prêtèrent aucune attention aux sondages qui avaient prédit correctement le résultat. Le modèle de Kuran permet encore de comprendre les déformations de l'image de l'opinion dans les régimes totalitaires, lesquels provoquent normalement une falsification des préférences. Il explique aussi la faiblesse de l'opposition contre le régime des castes en Inde: craignant de perdre les maigres avantages qu'ils tirent du système, les intouchables eux-mêmes manifestent souvent des comportements de réprobation voire de rétorsion à l'égard de ceux d'entre eux qui osent exprimer des critiques à l'encontre de la condition qui leur est faite.

On peut noter (ce que ne fait pas Kuran) que cette théorie de type B généralise la théorie de type A proposée par Tocqueville sur la déchristianisation du temps de la Révolution française. Il peut fort bien se faire qu'une majorité de gens croient blanc et que l'opinion publique paraisse croire noir, suggère Tocqueville. Cela se

8 Olson M., La logique de l'action collective, Paris, PUF, 1978.

29 Kuran T., Private Truths, Public Lies. The social consequences of preference falsification, Cambridge, Mass., Harvard U. Press, 1995, et ma discussion in Contemporary Sociology, 24, 6, 1996, 732-733. 
produit lorsqu'il est mal vu de déclarer qu'on croit blanc. Ainsi, au moment de la Révolution, l'opinion publique passait pour antireligieuse, alors qu'une forte majorité de la population conservait l' «ancienne foi». Mais comme elle ne jugeait pas opportun de s'exprimer, chacun des croyants avait l'impression qu'il était le seul à y rester fidèle. "Ceux qui niaient le christianisme élevant la voix et ceux qui croyaient encore faisant silence, il arriva ce qui s'est vu si souvent depuis parmi nous, non seulement en fait de religion, mais en tout autre matière. Les hommes qui conservaient l'ancienne foi craignirent d'être les seuls à lui rester fidèles, et, redoutant plus l'isolement que l'erreur, ils se joignirent à la foule sans penser comme elle. Ce qui n'était encore que le sentiment d'une partie de la nation parut ainsi l'opinion de tous, et sembla dès lors irrésistible aux yeux mêmes de ceux qui lui donnaient cette fausse apparence $»^{30}$. De même, on a cru que les Russes étaient en majorité devenus communistes, alors que seule une minorité avait adhéré à la foi nouvelle.

Mes propres travaux sur la frustration relative ${ }^{31}$ ou ma théorie des marchés intellectuels ${ }^{32}$, que Mendras évoque volontiers, sont des théories de type B. La seconde peut être raccordée au modèle de Kuran: dans les deux modèles, la cible visée tend à modifier le contenu du message qui lui est adressé. Le modèle des effets non intentionnels des actions intentionnelles (ou, plus brièvement, des effets d'agrégation ou des effets pervers), une notion classique que j'ai systématisée et illustrée à l'aide d'exemples inédits, peut également être inclus dans cette catégorie ${ }^{33}$.

Ces exemples démontrent que la sociologie contemporaine produit de façon plus ou moins continue des théories de type A et B. On a ici un mouvement cumulatif. Non seulement de nouveaux modèles se créent, mais on voit que des théories de type $\mathrm{A}$ inspirent des théories de type $\mathrm{B}$, lesquelles permettent à leur tour le développement de théories de type A. Ainsi, Kuran propose une vue nouvelle sur la persistance du régime des castes.

\section{Paradigmes (théories générales)}

Dans la famille des théories explicatives, certaines correspondent à un niveau d'abstraction encore plus grand. Peut-être le mot paradigme est-il approprié pour désigner ce type de théories. On peut les appeler aussi «théories de type C ». Elles entretiennent des relations de fécondation réciproque avec les théories des deux autres niveaux. On peut légitimement parler ici aussi de cumulativité, de scientificité et de progrès.

30 Tocqueville A. de, L'ancien régime et la révolution, in: Tocqueville. De la démocratie en Amérique, Souvenirs, l'Ancien Régime et la Révolution, Paris, Laffont, Coll. Bouquins, 1986, Livre III, Ch. 2, 1045.

31 Effets pervers et ordre social, Paris, PUF, Quadrige, 1993 (1977), ch.5.

32 Effets pervers, op. cit., ch.7, 222 sq.; «Les intellectuels et le second marché », Revue européenne des sciences sociales, XXVIII, 87, 1990, 89-103. «L'intellectuel et ses publics: les singularités françaises », in J.D. Reynaud et Y. Grafmeyer (éds.), Français qui êtes.vous? Des essais et des chiffres, Paris, La Documentation Française, 1981, 465-480.

33 Effets pervers, op.cit. Dans La place du désordre. Critique des théories du changement social, Paris, PUF, Quadrige, 1985 (1981), j'ai souligné que les changements sociaux doivent souvent s'analyser comme des effets de ce type. D'autres changements sont bien sûr des effets voulus. 
Le modèle du choix rationnel, le rational choice model des auteurs américains, est l'un de ces paradigmes. Il explique des phénomènes sociaux variés, comme le fait qu'on accepte sans sourciller maintes contraintes sociales. C'est pourquoi, de Rousseau à des sociologues contemporains comme Coleman ou Opp ${ }^{34}$, on analyse la genèse et la diffusion de certaines normes et valeurs à partir de ce paradigme. Il est vrai que ce modèle a un domaine de validité très ample. C'est bien parce que la situation serait pire sans eux qu'on accepte aussi facilement la contrainte sociale banale que représentent les feux de la circulation. Comme l'ont montré Buchanan et Tullock ${ }^{35}$, c'est parce qu'elle minimise la somme de coûts variant en sens opposé qu'on accepte aussi facilement de se plier à la règle de la majorité. Sans doute est-elle née au sein des couvents médiévaux ${ }^{36}$. Mais si elle a été socialement sélectionnée, c'est qu'elle fait sens pour les acteurs sociaux pour les raisons qu'indiquent Buchanan et Tullock. Or ces raisons sont du type de celles que considère le modèle du choix rationnel. Ce modèle ne s'applique d'ailleurs pas qu'à l'analyse des normes. Il est présent dans de nombreuses théories de type A, comme celle par laquelle Tocqueville explique le sous-développement de l'agriculture française à la fin du XVIIIe siècle ${ }^{37}$.

Mais, si le modèle du choix rationnel s'applique à de nombreux phénomènes, beaucoup d'autres lui échappent. Une expérience de psychologie sociale suffit à le montrer. On y propose à des sujets de jouer (une seule fois) au jeu dit « de l'ultimatum »: 1000 francs sont à partager entre $\mathrm{A}$ et $\mathrm{B}$. A fait une proposition de partage. B peut la prendre ou la laisser, mais non émettre une contre-proposition. Si $\mathrm{B}$ refuse la proposition de $\mathrm{A}$, les $1000 \mathrm{~F}$ restent dans la poche de l'expérimentateur. Cette expérience crée donc une situation asymétrique donnant à $\mathrm{A}$ un pouvoir de proposition et à $\mathrm{B}$ une simple capacité d'acceptation ou de refus. Si les joueurs obéissaient à des mobiles purement égoïstes et utilitaristes du type de ceux qu'envisage le modèle du choix rationnel, on pourrait s'attendre à ce que des propositions de type «700 francs pour moi (A) et 300 francs pour toi (B)» soient fréquemment observées. En fait, la proposition la plus courante est celle du partage égal. Ce résultat contredit bien l'axiome selon lequel l'acteur obéirait exclusivement à des raisons de type coûts-avantages. C'est pour rendre compte de ce type d'observations que Weber a proposé sa célèbre notion de la rationalité axiologique. Mais elle est loin d'être claire. De surcroît, la distinction entre rationalité axiologique et rationalité instrumentale ne répond pas, en elle-même, à la question de savoir pourquoi l'individu obéit tantôt à l'une et tantôt à l'autre. Or son choix à cet égard n'est généralement pas ad libitum. Au contraire, certaines situations impliquent socialement l'une des formes de rationalité, comme on le voit au fait que, comme dans le jeu de l'ultimatum, tous tendent à se rallier à la rationalité axiologique. Les catégories weberiennes invitent donc à élaborer une théorie qui les subsume.

34 Coleman J., Foundations of social theory, Cambridge, Harvard U. Press, 1990. Opp K. D., Die Entstehung sozialer Normen, Tübingen, Mohr, 1983.

35 Buchanan J. et Tullock G., The Calculus of Consent, Ann Arbor, U. of Michigan Press, 1967.

36 Moulin L. «Les origines religieuses des techniques électorales et délibératives modernes », Revue internationale d' histoire politique et constitutionnelle, avril-juin 1953, 106-148.

37 Sans affirmer en aucune façon l'universalité du modèle du choix rationnel, j'en ai abondamment illustré l'importance dans La logique du social, Paris, Hachette, Coll. Pluriel, 1983 (1979). 
L'insuffisance du modèle du choix rationnel provient de ce que beaucoup de comportements sont inspirés par des croyances positives et normatives. Or les croyances elles-mêmes ne s'expliquent par des considérations de coûts-avantages que dans des cas particuliers. Certaines traditions, comme les traditions marxiste ou nietzschéenne, font sans doute des croyances la manifestation inconsciente d'intérêts individuels ou collectifs. Mais, comme il est impossible d'accorder une validité générale à cette conception, elle ne peut assurer la généralité du modèle du choix rationnel.

Une analyse classique, de nouveau empruntée à Tocqueville, permet de concrétiser ce point essentiel. Pourquoi les intellectuels français croient-ils aussi fermement à la Raison à la fin du XVIIIe siècle, se demande-t-il dans L'Ancien Régime? La question a le statut d'une énigme, car le même phénomène ne se produit à la même époque ni en Angleterre ni aux Etats-unis. En fait, nous dit Tocqueville, ces intellectuels ont des raisons de croire à la Raison. Mais elles ne sont en aucune façon du type de celles qu'évoque le modèle du choix rationnel. On peut les qualifier de cognitives. La France de ce temps est dans un état tel que la tradition paraît à beaucoup comme la source de tous les maux. Les classes liées à la tradition, la noblesse et le clergé, sont discréditées. La première se tient à l'écart des affaires politiques locales et de la vie économique. Ceux des nobles, souvent désargentés, qui restent à la campagne sont d'autant plus accrochés à leurs privilèges qu'ils sont économiquement plus démunis: c'est pourquoi on les désigne par le nom d'un petit oiseau de proie, le hobereau. Le haut clergé ne se soucie que de paraître à Versailles. Le bas clergé est perçu comme ayant partie liée avec le haut et par là avec le pouvoir. Quant au pouvoir lui-même, il paraît peu attentif aux intérêts du peuple. De tout cela, se dégage le sentiment que les traditions sont mauvaises, que les institutions sont dépourvues de fonction et qu'elles représentent des vestiges d'un passé révolu, que les distinctions de rang sont injustifiables et par suite illégitimes, et que le statut des personnes est déconnecté de leur importance sociale. Une équation s'établit alors: Tradition $=$ Chaos $=$ Inégalité $=$ Injustice $=$ Mal et par opposition Raison $=$ Planification $=$ Ordre $=$ Équité $=$ Justice $=$ Bien. D'où la vision artificialiste des sociétés que développeront les philosophes des Lumières, et leur ardeur à rebâtir la société à partir de plans conçus selon la Raison: «tous pensent qu'il convient de substituer des règles simples et élémentaires, puisées dans la raison et la loi naturelle, aux coutumes compliquées et traditionnelles qui régissent la société de leur temps» $(. . .)^{38}$. Tocqueville n'a qu'aversion pour cette manière de voir. Mais il n'interprète à aucun moment ces croyances comme dénuées de sens. Au contraire, le contexte français fait que les intellectuels ont de bonnes raisons d'y croire, tout comme leurs homologues anglais ont de bonnes raisons de ne pas y croire ${ }^{39}$. Derrière la croyance à la Raison

Tocqueville, op. cit., Livre III, Ch.1, 1036.

39 Par «bonnes raisons », j'entends les raisons qui dans le contexte de l'acteur sont bonnes, même si elles sont jugées fausses par l'observateur. Les physiciens du XIXe siècle ont de bonnes raisons, dans le contexte cognitif qui est le leur, de croire à l'hypothèse de l'éther, raisons que nous n'avons plus et qui font que l'hypothèse est jugée fausse aujourd'hui. On peut définir la notion de bonne raison de façon analytique comme caractérisant les argumentations comportant au moins un énoncé localement (i.e. contextuellement) valide. Bien entendu, l'acteur dépourvu par définition de possibilité de distanciation peut ne pas voir le caractère contextuel de tel énoncé. La notion de 
et la disqualification de la Tradition, Tocqueville décèle en d'autres termes un argumentaire qui en fonde le sens pour chaque individu et explique ainsi qu'elle ait pu devenir collective.

Tocqueville résout ici brillamment un difficile problème de sociologie de la connaissance. Il le doit au paradigme qu'il utilise: celui-ci part du principe que les croyances collectives s'installent lorsqu'elles s'appuient sur des raisons perçues comme fortes par les acteurs sociaux dans le contexte qui est le leur. J'ai essayé de codifier ce paradigme et de montrer qu'il avait une portée générale dans L'idéologie, puis, de façon plus approfondie, dans L'art de se persuader ${ }^{40}$. Les meilleures analyses de la sociologie de la connaissance relèvent de ce paradigme. Mais bien d'autres phénomènes, comme les dérapages de l'inférence naturelle qu'étudie la psychologie cognitive, peuvent aussi s'expliquer dans ce cadre. Les résultats de la psychologie cognitive sont particulièrement intéressants pour la sociologie, car les mécanismes générateurs des croyances collectives sont souvent plus faciles à discerner in vitro qu'in vivo.

Bien des difficultés soulevées par l'explication des croyances collectives de type normatif peuvent également être résolues grâce à ce paradigme ${ }^{41}$. Ainsi, on observe que les sujets sociaux apparaissent dans certains cas comme indifférents, dans d'autres comme très attentifs à l'application du principe de l'égalité rétribution-contribution. On a bien là une énigme et, de surcroît, une énigme scientifiquement stratégique, puisqu'elle suffit à disqualifier les théories, qui connaissent une certaine vogue aujourd'hui, selon lesquelles les sentiments moraux s'expliqueraient à partir de données inscrites dans la nature humaine. Ces théories ne permettent pas en effet de comprendre pourquoi la violation du même principe peut selon les cas provoquer ou non une réaction de désapprobation. La même énigme révèle l'insuffisance d'un autre paradigme, par ailleurs très puissant, la théorie de l'échange, qui explique bien l'importance dans la vie sociale du principe de l'égalité rétribution-contribution, mais non les modulations complexes que les acteurs sociaux lui font subir.

Le fonctionnalisme représente, dans ses meilleures versions, un autre exemple de paradigme très utile s'agissant de la théorisation des phénomènes normatifs. Dans son principe, il pose qu'une situation est considérée comme bonne par les individus lorsqu'elle facilite le fonctionnement d'un système auquel ils sont attachés. Ainsi, les membres d'un club admettront qu'on en contrôle l'entrée, cette disposition étant indispensable si l'on veut écarter les candidats peu motivés ou incapables de contribuer au bon fonctionnement du club. C'est pourquoi les académies ou les clubs sportifs sélectionnent leurs membres. Mais le même exemple révèle aussi les limites du fonctionnalisme, qui n'explique pas pourquoi on admet si difficilement que soit contrôlée la sortie d'un club. En restreignant la sortie, on risque certes de retenir des membres qui n'éprouvent plus d'intérêt pour les activités du club et, par là, de nuire à son fonctionnement. Mais, comme on le voit à

«bonne raison» suppose en d'autres termes qu'on puisse se donner un observateur distancié capable de voir le caractère contextuel de l'argumentation de l'observé.

40 L'idéologie, ou l'origine des idées reçues, Paris, Fayard/Seuil, Coll. Points, 1992 (1986). L'art de se persuader, Paris, Fayard/ Seuil, Coll. Points, 1992 (1990).

${ }^{41}$ Le juste et le vrai : études sur l'objectivité des valeurs et de la connaissance, Paris, Fayard, 1995. 
l'exemple des sectes, interdire la sortie d'un club est jugé non seulement inopportun, mais moralement choquant. Or ce n'est certainement pas par souci du bon fonctionnement des sectes qu'on réprouve qu'elles retiennent leurs membres. La réaction morale est ici le produit de raisons fortes, mais ne relevant pas des catégories qu'envisagent le modèle du choix rationnel, le fonctionnalisme ou la théorie de l'échange.

La critique, au sens kantien, du corpus d'observations que nous rapportent la sociologie, la psychologie sociale ou tout simplement la vie courante à propos des sentiments moraux, m'a finalement conduit à en proposer dans Le juste et le vrai une théorie «cognitiviste». Elle s'applique aussi bien aux croyances normatives universelles qu'aux croyances contextuelles. Car, n'en déplaise aux culturalistes, il existe bel et bien des croyances universelles. Il est entendu qu'on coupe la main des voleurs dans certaines sociétés et qu'on les laisse plutôt courir dans d'autres, que la politesse veut tantôt qu'on ôte son chapeau et tantôt qu'on le garde; mais le vol et l'impolitesse font l'objet d'une condamnation universelle. Dans d'autres cas, les raisons qui sous-tendent les croyances normatives ne sont pas universalisables et sont au contraire dépendantes du contexte. Cette double remarque m'a amené à parler d' «axiologie post-newtonienne» à propos de cette théorie cognitiviste ${ }^{42}$.

Elle contient la théorie de l'échange, le fonctionnalisme et le modèle du choix rationnel comme des cas particuliers. Cette généralité lui permet d'expliquer maints phénomènes énigmatiques en dehors de ceux que j'ai déjà évoqués. Une belle étude de caractère quasi-expérimental révèle par exemple que les principes de justice de Harsanyi et plus encore de Rawls apparaissent comme incompatibles avec l'intuition morale du public ${ }^{43}$. Pourquoi en est-il ainsi? Autres questions: d'où provient l'évolution de la sensibilité morale? Relève-t-elle de la seule contingence? Pourquoi dans certaines situations la justice est-elle définie par l'équité, dans d'autres par l'égalité? Cette théorie permet aussi de clarifier le difficile problème de la nature de la contrainte morale. Pareto note, sur un ton sarcastique, que nous disons volontiers «X est bien» là où, selon lui, il faudrait dire «je crois que $X$ est bien». Cette remarque condense une importante théorie de l'évaluation morale qui a été souvent développée, sous des formes diverses: l'élision en question indiquerait que nous nous auto-illusionnons; nous croyons que nos croyances morales sont objectivement fondées, alors qu'elles sont l'expression de sentiments que nous cherchons à nous dissimuler à nous-mêmes. En fait, il est inutile de supposer qu'il y ait ici élision. Le sujet ne peut endosser une croyance morale que s'il a l'impression qu'il a des raisons de le faire. Or une raison n'est telle pour lui que si elle revêt à ses yeux une valeur objective, impliquant que l'Autrui généralisé devrait aussi la partager.

La théorie cognitiviste pose en conséquence que la contrainte que nous éprouvons à croire que «X est bien» est de même ordre que celle qui nous fait croire que «X est vrai». C'est pourquoi croyances collectives normatives et positives peuvent être analysées à l'aide du même paradigme.

42 J'en ai proposé une présentation ramassée dans le Sidney Ball Lecture sur «The moral sense » que j'ai été invité à donner à Oxford le 20 novembre 1995. Voir aussi Le sens des valeurs, Paris, PUF, Quadrige, 1999.

43 Frohlich, N et Oppenheimer, J.A., Choosing justice, an experimental approach to ethical theory, U. California Press, Los Angeles /Oxford, 1992. 
Cette théorie permet en fin de compte de résorber les difficultés soulevées par les théories des sentiments moraux actuellement disponibles. Bien qu'elle ait été esquissée et appliquée instinctivement par Weber et quelques autres, je ne crois pas qu'elle ait été proposée jusqu'ici dans les termes où je le fais. Elle contredit en effet une idée reçue dont l'influence est forte, bien qu'elle soit en général inconsciente, celle de l'existence d'un gouffre entre le positif et le normatif.

On voit en tout cas qu'il existe dans la sociologie d'aujourd'hui une prolifération de paradigmes et une confrontation de ces paradigmes visant à déterminer le plus fécond, le plus puissant, le plus général, celui qui représente le meilleur compromis entre les nécessités de la simplification et celles du réalisme. Une telle discussion n'est en rien différente de celles qu'on rencontre dans les sciences de la nature ${ }^{44}$.

J'ai omis de souligner que, derrière toutes les théories de types $\mathrm{A}, \mathrm{B}$ et $\mathrm{C}$ que j'ai évoquées, on détecte facilement les deux principes de l'individualisme méthodologique: les actions et/ou croyances collectives résultent de l'agrégation d'actions et/ou croyances individuelles; les raisons que les acteurs ont d'endosser telle croyance ou d'entreprendre telle action sont les causes de cette croyance ou de cette action. Lorsqu'on précise que les raisons en question relèvent du registre coûts-avantages, on retrouve la version très particulière de ce paradigme que représente le «modèle du choix rationnel» au sens des auteurs américains ${ }^{45}$.

Enfin, on aura compris que les types de théories que je distingue ici sont réels, me semble-t-il, mais flous, comme le sont par nature les types-idéaux. Cette remarque me conduit de plain pied à mon point suivant.

\section{Typologies}

Bien que la production de théories représente sans aucun doute la dimension essentielle de l'activité scientifique de la sociologie, elle ne s'y réduit pas. Importante est aussi la production conceptuelle. Un exemple classique ici est celui des types d'autorité de Max Weber. La sociologie moderne en fournirait facilement bien d'autres. Je me contenterai d'en évoquer un. Récemment, une sociologue québécoise s'est attaquée avec originalité à un sujet labouré, le suicide ${ }^{46}$. Elle part de l'hypothèse que les suicides de jeunes, dont la montée est spectaculaire dans les dernières décennies au Québec, sont surtout des «suicides d'être », c'est-à-dire des suicides témoignant d'un échec soit à se donner des valeurs, soit à mobiliser

44 Voir la discussion à laquelle ont participé J. Agassi, H. Albert, E. Ben Rafael, F. Crespi, F. Fuchs, H. Klimt, P. Legrenzi et R. Rumiati, S. Lindenberg, S. Lukes, M. Mondadori, A. Montesano, R. Nadeau, A. Pizzorno, S.A. Salvaggio et T. Vandevelde, International studies in the philosophy of science, vol. 7, $\mathrm{N}^{\circ} 1,1993,21-86$ sur mon article "Toward a synthetic theory of rationality », Ibid., 5-19. Voir aussi ma réponse: «More on 'good reasons': reply to critics », Ibid., vol. 7, n 1 , 1993, 87-102.

45 J'ai développé ce point in «Individualisme et holisme dans les sciences sociales », in: Birnbaum P. et Leca J. (éds), Sur l'individualisme, Paris, Presses de la Fondation Nationale des Sciences Politiques, 1986, chapitre 2, 45-59, et «The individualistic tradition in sociology », in Alexander J. C., Giesen B., Münch R., Smelser N. J. (eds), The micro-macro link, Berkeley, U. of California Press, 1987, 45-70.

46 Gratton Jacob F., Les suicides d' «être» de jeunes québécois, Sainte-Foy (Québec), Presses de l’Université du Québec, 1997. 
les ressources appropriées pour réaliser les valeurs qu'on s'est données. Elle oppose ces suicides d'être aux «suicides d'avoir», caractéristiques d'âges plus avancés et qui résultent d'une difficulté qui atteint l'individu dans son rapport au passé (par exemple perte d'un être cher), et aussi aux «suicides d'émotion», qui traduisent des difficultés de l'individu dans son rapport au présent. Au cours d'un grand nombre d'heures d'entretien avec des témoins, elle a alors recueilli le matériau de base qui lui a permis de reconstituer cinq histoires de vie de jeunes suicidés. Celles-ci s'articulent effectivement autour des deux pôles «valeurs » et «ressources». L'un des cas (le $\mathrm{n}^{\circ} 1, \mathrm{l}^{\prime}$ «idéaliste») se donne des valeurs dont il a l'impression qu'il ne peut les atteindre, non qu'il ne dispose pas de ressources de tous ordres, mais parce que son niveau d'exigence est très élevé. Un autre des cas (le $\mathrm{n}^{\circ} 2$, le «blasé») n'arrive pas à se donner des valeurs, en dépit de ressources importantes. Le 3e cas (le «nostalgique») se donne une sorte d'âge d'or personnel qu'il ne peut retrouver. Le 4e cas (l' «épuisée») se laisse happer par un engrenage qui finit par le laisser dépourvue de ressources. Un autre cas (le $n^{\circ} 5$, la « déshéritée») manque de ressources et n'est pas à même de se donner des valeurs.

La dimension conceptuelle de l'activité sociologique n'a jamais été bien étudiée. Cela est regrettable. Certains de ces concepts que Weber jugeait propres à la sociologie et qu'il qualifiait d' «idéal-types» sont efficaces ${ }^{47}$. Ainsi, on a l'impression que les types de suicide que je viens d'évoquer correspondent bien à des réalités. En revanche, d'autres paraissent plus insaisissables et par suite d'utilité plus incertaine. On en a fait la démonstration à propos du concept d'anomie par exemple $^{48}$ et l'on peut, plus généralement, émettre des doutes sur la réalité des types de suicide que distingue Durkheim. D'autres concepts, comme les termes de l'opposition entre sociétés traditionnelles et sociétés modernes, ont été indéfiniment déclinés. Mais représentent-ils plus que des guides sommaires? D’autres créations conceptuelles sont de caractère impressionniste et ont surtout une valeur suggestive, comme celles qui déclarent nos sociétés tribales, réflexives ou complexes, ou celles qui opposent les sociétés dionysiaques aux sociétés apolliniennes.

\section{Données surprenantes}

Ce catalogue n'épuise évidemment pas les contributions de la sociologie à la connaissance du social. Quelquefois, le sociologue recueille des données surprenantes, qui ont un grand intérêt en elles-mêmes, comme lorsque Girod montre que l'année de naissance n'a pas moins d'influence sur les chances devant la vie que l'origine sociale ${ }^{49}$. À elle seule, cette observation bouscule bien des idées reçues. Les données différentielles sur la religiosité posent, de même, des problèmes fascinants. On observe que les Scandinaves sont les plus athées et que les Américains

47 Ces invariants transhistoriques, essentiels puisqu'ils constituent la source principale de notre intérêt pour l'histoire, ont inspiré la «sociologie formelle» de Simmel comme les «types-idéaux » de Weber. L'exploitation de cette idée a été reprise avec bonheur par Rezsohazy R., Comprendre l'action et le changement politiques, Bruxelles, de Boeck, 1996.

48 Besnard P., L'anomie, Paris, PUF, 1987.

49 Girod R., Inégalité, inégalités, Paris, PUF, 1977. 
restent aujourd'hui les plus religieux des Occidentaux ${ }^{50}$. Ici encore, il s'agit de données inaccessibles à la sociologie spontanée. Non seulement de telles données sont porteuses d'information, et invitent à la réflexion et à l'explication, mais elles ont une vertu critique. Elles permettent notamment de mesurer la grossièreté des théories de la modernité.

En revanche, on ne nous apprend pas grand'chose quand on nous révèle, corrélation poussée jusqu'à la n-ième décimale à l'appui, que les cadres supérieurs envoient plus souvent leurs enfants à l'École Polytechnique que les plombiers-zingueurs. Mais il ne faut pas tenir rigueur à la sociologie des données inutiles qu'elle produit. Tout d'abord, on n'est jamais sûr de ne pas trouver une perle dans l'enquête la plus banale. Ensuite, des données même triviales peuvent satisfaire des passions sociales, voire exercer une action cathartique. Et puis, recueillir des données soi-même, c'est s'assurer d'un droit de propriété inaliénable sur une recherche: mon pays n'est pas le plus beau, mais c'est mon pays.

Mais réduire la sociologie aux travaux d'enquête, c'est la mutiler.

\section{Techniques}

La sociologie progresse aussi sur le plan des techniques, notamment de collecte et d'analyse des données. Mais les percées techniques sont surtout intéressantes lorsqu'elles ont un impact méthodologique (d'où l'importance de la distinction entre méthodologie et techniques). Les travaux de Gini sur la mesure des inégalités ont mis en évidence que le concept apparemment le plus simple, celui d'inégalité, était en fait très complexe. Ils ont ouvert la voie à une série de recherches démontrant qu'il ne peut y avoir de mesure absolue des inégalités (qu'il s'agisse des inégalités de revenu ou d'autres formes d'inégalités). La mobilité sociale a donné lieu à une réflexion analogue, d'où il ressort qu'il y a plusieurs manières de la mesurer, ces mesures ne se ramenant pas les unes aux autres. J'ai moi-même contribué à cette discussion et montré qu'on pouvait définir un indice alternatif de l'indice de Yasuda, tout aussi légitime que lui, et pouvant en principe donner une réponse contradictoire avec la sienne à la question de savoir si la mobilité est plus grande ou moins grande aujourd'hui qu'hier, ou si elle est plus ou moins importante dans telle société que dans telle autre ${ }^{51}$.

Il faut relever ici que la technique peut revêtir une fonction surtout symbolique. Des banalités issues d'une analyse factorielle passeront facilement pour des révélations, voire des découvertes. Sainte-Beuve a remarqué avec humour que ses écrits ont été mieux accueillis du jour où il est rentré à l'Académie française sans, précise-t-il, que leur valeur ait changé en quoi que ce soit de ce fait. Une idée peut de même être perçue plus favorablement dès lors qu'elle apparaît légitimée par des signes extérieurs de scientificité, sans être plus juste ou plus intéressante pour autant.

50 Bell D., «The disunited states of America, middle class fears turn class war into culture wars», Times literary supplement, june 9, 1995, 16-17.

51 «A note on social immobility and inequality measurement», Quality and Quantity, VI, 1, 1972, 17-35. 


\section{AUTRES FONCTIONS DE LA SOCIOLOGIE}

Il n'y a donc guère de doute que, comme toute science, la sociologie est capable de créer du savoir. Elle produit aussi, bien sûr, des gaspillages et du faux savoir. Par exemple: la théorie selon laquelle les valeurs esthétiques ne seraient qu'une projection des opinions des mondes de l'art ${ }^{52}$, ou celle selon laquelle les croyances scientifiques seraient dépourvues de fondement objectif. Mais l'arbre ne doit pas cacher la forêt. L'état des sciences sociales n'explique pas le scepticisme ambiant.

On ne peut comprendre ce scepticisme si on ne perçoit pas, j'y insiste à nouveau, que cette veine scientifique n'est qu'un élément dans un magma. Je veux dire que la sociologie poursuit des objectifs autres que scientifiques et, ajouteraije, elle les poursuit naturellement. Un bref inventaire me paraît utile ici pour compléter mon exercice de sociologie de la connaissance et répondre à la question «Pourquoi ce scepticisme?» ${ }^{53}$.

La sociologie assume souvent une fonction descriptive à finalité caméraliste. Il s'agit alors pour elle de faire remonter à un niveau central le savoir détenu par les individus et généralement de réunir des informations diverses utiles à la décision. Ainsi, lorsqu'un Le Play observa méthodiquement le mode de vie des ouvriers européens $^{54}$, il rendit accessibles à tous des informations évidemment familières aux individus eux-mêmes, et permit ainsi aux gouvernants de l'époque de préciser les politiques sociales qu'ils cherchaient à mettre en œuvre. De même, la lutte contre le crime ou contre le SIDA est rendue plus efficace aujourd'hui par les enquêtes sociographiques portant sur ces phénomènes. Cette fonction d'information est importante: elle intéresse les gouvernements, les syndicats, les mouvements sociaux, les mouvements de pensée, toutes les institutions possibles et imaginables, ainsi que les medias et le public lui-même.

Dans d'autres cas, la sociologie se présente plutôt comme l'héritière de la philosophie de l' histoire. Cette discipline, dont on fait généralement remonter l'existence à l'Essai sur les mours de Voltaire, vise à faire apparaître les régularités et les tendances qui rythment le cours de l'histoire. Les théories dites de la modernité, les essais qui s'efforcent de décrire les forces souterraines qui travaillent les sociétés ou de débusquer les mouvements sociaux porteurs d'avenir, sont illustratifs de ce type de sociologie. Il ne s'agit pas ici d'expliquer des phénomènes énigmatiques, mais de réduire l'inquiétude et si possible l'incertitude face à l'avenir.

La sociologie peut encore chercher à exercer une influence directe sur les institutions sociales. Elle a alors une fonction qu'on peut qualifier de performative ou de critique (au sens dans ce cas, non de Kant, mais de l'école de Francfort). Ainsi, la théorie de la stigmatisation ${ }^{55}$ ne représente certainement pas une percée scienti-

52 Voir ma critique de cette théorie in: «De l'objectivité des valeurs artistiques ou les valeurs artistiques entre le platonisme et le conventionnalisme», Archives de philosophie du droit, 1995, 40, 76-95.

53 Je reprends ici en partie l'argument de mon article «Comment écrire l'histoire des sciences sociales?», Communications, 54, 1992, 299-317.

54 Le Play F., Les ouvriers européens, (1855), Paris, Hachette, 1971.

55 Goffman E., Stigmate, les usages sociaux des handicaps, trad. de Stigma (1963), Paris, Minuit, 1975 . 
fique comparable à la théorie des interférences de Fresnel. Chacun sait que le fait par exemple de sortir de prison «stigmatise» le candidat à l'emploi et la sociologie spontanée n'avait pas attendu Goffman pour relever ce type de phénomène. Mais en le plaçant sous l'autorité de la science, il l'épinglait et contribuait à le constituer en "problème», au sens politique du mot.

La sociologie exerce aussi parfois ce qu'on peut appeler une fonction littéraire. Aujourd'hui, sans le dire aussi expressément, certains courants de pensée lui proposent même d'essayer de concurrencer la littérature. Il est vrai que les grandes œuvres littéraires contiennent souvent de précieuses analyses sociologiques. Dans Un cour simple, Flaubert présente une analyse des relations entre maître et servante propre à faire pâlir d'envie «phénoménologues » (au sens un peu particulier que les sociologues donnent à ce terme), «ethnométhodologues » et autres «interactionnistes symboliques »: Madame ne comprend pas que Félicité fasse état devant elle de la peine que lui cause la maladie de son neveu; la distance qui la sépare de sa servante est si grande que les soucis de cette dernière ne peuvent par principe la concerner; comment Félicité peut-elle faire passer ses sentiments, aussi compréhensibles soient-ils, avant cette évidence sociale? Ce passage merveilleux illustre peut-être ce que C. Geertz appelle la thick description. En un temps qui ne produit plus guère de Flaubert, la sociologie tente quelquefois de reprendre, avec ses moyens, le flambeau de la littérature. Un charmant collègue me disait récemment avoir été ébloui par la «découverte» d'un sociologue à la mode révélant qu'un compliment peut avoir une valeur minorante. Molière et Shakespeare l'avaient fort bien dit depuis longtemps, me semble-t-il. Mais ce texte passait auprès de ce collègue pour l'œuf de Christophe Colomb et il y voyait la preuve que les Américains ont bien raison de traiter tout compliment adressé par un homme à une femme comme le signe d'un sexisme coupable.

Enfin, certains travaux sociologiques se distinguent mal des reportages journalistiques, des enquêtes historiques et de productions diverses n'ayant rien de spécifiquement sociologique.

Cet inventaire n'est pas exhaustif, mais il suffit à souligner le polymorphisme quasi naturel de la sociologie. Ses fonctions non scientifiques ne sont évidemment pas dénuées d'importance sociale, loin de là. Mais il faut remarquer derechef que si Weber, Pareto, Tocqueville ou Durkheim sont placés d'un commun accord au premier rang, c'est qu'ils ont apporté des contributions majeures dans un registre proprement scientifique, plutôt que dans ces registres annexes. En tout cas, le polymorphisme de la sociologie, qui s'est accentué ces dernières années sous l'effet de divers facteurs, comme l'extension de la demande de type caméraliste ou la diffusion du relativisme, explique pour une part les doutes des sociologues d'aujourd'hui sur l'identité de leur discipline. Signe de cette évolution: déjà, sur les rayons des librairies l'étiquette «Questions de société» commence à remplacer «Sociologie».

\section{POURQUOI JE SUIS DEVENU SOCIOLOGUE}

Je terminerai par une note personnelle sur mes années d'apprentissage. Je me suis toujours reconnu dans une conception scientifique de la sociologie. Cette préférence peut être rattachée à certaines expériences de sympathie ou d'antipathie 
intellectuelle datant du temps de mes études supérieures. Ces sentiments étaient eux-mêmes liés à l'impression de clarté ou au contraire d'opacité que j'ai éprouvée à la lecture de tel ou tel auteur. Je crois n'avoir jamais eu de difficulté à comprendre une phrase de Stoetzel, Lazarsfeld, Homans, Cazeneuve, Crozier, Dahrendorf ou Merton ni, du coté des auteurs classiques, de Tocqueville, Simmel, Weber ou Halbwachs. J'ai toujours eu l'impression de circuler dans l'univers mental de ces auteurs (et d'autres) avec aisance, de comprendre la nature de leurs projets, et aussi de m'instruire en les lisant, même lorsque je me sentais en désaccord avec eux. En revanche, j'ai toujours eu de la peine à pénétrer le monde d'autres auteurs. J'ai lu très tôt Parsons, qui fut considéré comme l'un des papes de la sociologie, et j'ai toujours éprouvé une grande déférence pour sa ténacité et son intégrité intellectuelles. J'étais sensible au fait qu'il plaçât la notion d'action au centre de ses constructions. En même temps, il me semblait avoir une conception particulière de l'activité théorique. Plutôt que d'expliquer des phénomènes sociaux bien circonscrits, il me paraissait se soucier surtout de forger des systèmes de concepts dont il espérait qu'ils se révéleraient indispensables à l'analyse sociologique. Or il me semblait évident que la théorisation ne se réduit pas à la création de concepts. Mais la connivence intellectuelle n'est pas un ingrédient obligatoire de la sympathie personnelle. Je me souviens avec plaisir des déjeuners que j'ai partagés avec lui à Harvard. Dans ce milieu un peu puritain, il était le seul à se permettre un verre de vin. De passage à Paris la semaine qui précéda sa mort, il m'a offert un exemplaire dédicacé de son dernier livre. J'y ai été très sensible.

J'ai ressenti pour d'autres auteurs une antipathie immédiate, notamment pour ceux qui voient le sujet social comme muni d'une conscience fausse par essence. Ils se donnent ainsi le droit d'écarter tous les faits de conscience (des autres) et le privilège d'échapper eux-mêmes à la fausse conscience. Les vulgates marxienne, nietzschéenne ou freudienne ont entraîné Foucault et des auteurs plus mineurs dans ce piège. Mais cette antipathie ne s'est transformée en rejet que lorsqu'elle était à la fois intellectuelle et morale. J'ai éprouvé ce type de sentiments à l'égard par exemple de Le Bon. Dans une critique en forme d'épigramme dont la lucidité, la portée et aussi la qualité d'écriture méritent d'être soulignées, Stoetzel a dit de lui ${ }^{56}$ : «il lui faut l'automatisme et la contagion». Ces notions-clés de la sociologie de Le Bon rappellent en effet furieusement la «vertu dormitive» de Molière (dont on trouverait d'autres illustrations dans la sociologie d'aujourd'hui). Mais ce qui a engendré mon aversion à son endroit, c'est son côté imposteur. Il avait suffisamment de talent pour séduire un public peu critique en alimentant ses passions, mais il ne nous a rien appris. Malgré cela, il prétendait et était parvenu à créer auprès des demi-habiles l'illusion qu'il inaugurait une scienza nuova. Il existe deux types d'imposteurs: les séducteurs et les manipulateurs. Le séducteur est un affamé de popularité, le manipulateur un passionné d'influence. J'ai toujours été choqué par le mépris que l'un et l'autre vouent à ceux qu'ils subjuguent. Toute époque a ses Le Bon et la nôtre a les siens. Il ne faut commencer à s'inquiéter que lorsqu'ils réussissent à passer pour des autorités scientifiques.

C'est également pour des raisons morales que, très tôt au cours de mes études supérieures, j'ai cessé de me ressentir comme de gauche, sans tout d'abord m'en

56 Stoetzel J, Psychologie sociale, Paris, Flammarion, 1978 (1968). 
rendre clairement compte. J'en ai décrypté la raison par la suite: ce changement provenait de ce que j'avais éprouvé progressivement de plus en plus de difficulté à accepter l'assurance avec laquelle certains intellectuels de gauche se voyaient comme les dépositaires exclusifs de la conscience universelle, s'attribuaient le monopole à la fois de l'esprit, du cœur, du désir de progrès et de la générosité, et concluaient que cette supériorité pour ainsi dire substantielle les dispensaient, non seulement de toute discussion réglée de leurs propres idées, mais de toute considération pour autrui. Cette intolérance se manifestait de mille façons. Ainsi, du temps où j'étais à l'École Normale, la cellule communiste débattit sérieusement du point de savoir s'il fallait interdire aux camarades la lecture du journal $L e$ Monde, suspect à ses yeux de collusion avec la bourgeoisie. Mais cet état d'esprit était loin d'affecter les seuls communistes. Le prestige de Sartre, qui avait réussi à ériger la grossièreté et l'insulte en vertus ("Ayer est un con») a sans doute contribué à légitimer l'intolérance qui a miné la vie intellectuelle française de ces dernières décennies. Sociologiquement, l'installation de cette contre-valeur s'explique, sans pour autant bien sûr se justifier, par le fait que la barbarie qu'avaient engendrée les idéologies de droite avait placé les idéologies de gauche en position de monopole. Nous ne sommes qu'incomplètement sortis de cette situation, mortelle pour la pensée, où le «d'où parle-t-il?» et le «avec qui/contre qui est-il?» tiennent lieu de critique, même dans des organes réputés sérieux. Malgré le fracas qu'elle a entraîné, la chute du mur de Berlin a laissé intacts toutes sortes de petits murets.

Dès mes années de préparation à l'École Normale, la dissertation et les divers exercices rhétoriques du concours d'entrée ont provoqué chez moi un sentiment de malaise, parce qu'ils me paraissaient viser la séduction plutôt que la conviction $^{57}$. J'ai ressenti une honte diffuse en apprenant que j'avais obtenu la note de 17 sur 20 à l'épreuve orale de philosophie. Ironiquement, je dus ce succès à un exposé sur le thème de «l'échec». Plus tard, j'ai donné une «leçon» sur la psychanalyste Mélanie Klein dans le cadre de la préparation de l'agrégation. Notre répétiteur, L. Althusser, m'en a fait des compliments appuyés. Non seulement je n'y ai pas été sensible, mais ils m'ont gêné, car j'avais, ici encore, l'impression d'avoir séduit en disant à peu près n'importe quoi. Très tôt, j'ai été prévenu contre la verbosité et sensibilisé à cette stratégie classique de l'imposture qui consiste à faire passer l'obscurité, ce cache-misère de la platitude, pour de la profondeur. C'est pourquoi sans doute, une fois devenu sociologue, je me suis arrimé au principe que la sociologie n'est faite ni pour séduire ni pour influencer, mais pour éclairer.

Jules Renard a écrit dans son Journal que la clarté est la politesse de l'homme de lettres. Elle est aussi la marque de l'honnêteté de l'homme de science et de l'homme de pensée.

Me voici revenu au «Stoetzel envoie Boudon chez Lazarsfeld à Columbia» que je contestais au début de ces notes. Personne ne m'a envoyé à Columbia, mais j'avais décidé de faire un stage chez Lazarsfeld à l'issue de mon service militaire, parce que j'avais découvert un peu par hasard sur les rayons de la bibliothèque de la rue d'Ulm The language of social research. À tort ou à raison, ce livre m'était

57 Voir ma pochade sur ce sujet in: Peyrefitte A., Rue d'Ulm, Paris, Fayard, 1994, 528-31. 
apparu comme offrant une alternative à la sociologie gurvitchienne alors régnante en France, que j'avais toujours soupçonnée de dissimuler beaucoup de banalités dans des maquis de typologies et de définitions tatillonnes, et à sa concurrente structuraliste, qui me paraissait sujette à caution, tant par son ambition totalisante que par son platonisme ${ }^{58}$. L'introduction de l'ouvrage a tout de suite attiré mon attention par son insistance sur le fait que le progrès de la connaissance passe avant tout par la critique des produits de la recherche, et par sa défense de l' explication de texte comme outil essentiel de la pensée sociologique ${ }^{59}$. Cette idée ne m'a plus quitté depuis lors, comme on le voit aux nombreuses explications de texte qui parsèment mes travaux. J'ai formé à ce moment-là le projet d'un stage à Columbia. J'ai pu le réaliser grâce à une bourse que m'a obtenue Aron. Souhaitant être au clair sur ce sujet, j'ai décidé à la fin de mon séjour à New York de dresser un bilan des apports de la pensée mathématique aux sciences sociales et de le présenter comme thèse principale ${ }^{60}$. J'ai alors sollicité le patronnage de Stoetzel et ai fait sa connaissance à cette occasion. Bien plus tard, j'eus le bonheur d'avoir à prononcer publiquement son éloge ${ }^{61}$.

Mon intérêt pour le langage des sciences sociales explique non seulement le caractère théorique et méthodologique de nombre de mes contributions ${ }^{62}$, mais aussi mon nomadisme thématique : tout sujet m'a paru bon dès lors qu'il m'attirait en lui-même et présentait un intérêt théorique, qu'il relevât de la sociologie de l'éducation, de la mobilité, des inégalités, du changement social, de la justice, de la morale, des valeurs, de la connaissance, de la science, de l'art, des intellectuels ou des mouvements étudiants. A la réflexion, toutes mes recherches sont tout de même fédérées par un thème: celui de l'explication des croyances aux idées douteuses, fragiles ou fausses... et aussi aux idées vraies!

Ç'a été également mon choix exclusif - et un choix délibéré - de chercher à passer aussi rapidement que possible à l'Université plutôt que de rester au CNRS,

58 Voulant en avoir le cœur net, j'ai consacré ma thèse secondaire à ce sujet: À quoi sert la notion de structure? Essai sur la signification de la notion de structure dans les sciences humaines, Paris, Gallimard, 1968. Cette critique du structuralisme, alors à son apogée en France, a été surtout remarquée par sa traduction en anglais. Homans, grand sociologue, et homme bourru et quelque peu hautain (peut-être parce qu'il descendait en ligne directe du second président des États-Unis) aimait à en discuter avec moi. Bien des années après, C. Lévi-Strauss devait montrer indirectement, à travers sa présentation de L'art de se persuader devant l'Académie française, qu'il ne m'en tenait pas rigueur, Séance publique annuelle, Paris, Institut de France, 1991, $\mathrm{N}^{\circ} 18$.

59 Voir mon introduction aux morceaux choisis de Lazarsfeld dont l'Université de Chicago m'a confié la responsabilité, Paul F. Lazarsfeld: On social research and its language, Chicago, U. of Chicago Press, 1993 et aussi ma communication «L' «analyse empirique de l'action» de Lazarsfeld et la tradition de la sociologie compréhensive» au colloque «Paul Lazarsfeld» organisé à l'initiative de l'Ambassade d'Autriche à Paris, U. de Paris-Sorbonne, déc.1994, in J. Lautman et B.-P. Lécuyer (eds), Paul Lazarsfeld, Paris, L'Harmattan, 1998, 363-382.

60 L'analyse mathématique des faits sociaux, Paris, Plon, 1967.

61 «Notice sur la vie et les travaux de Jean Stoetzel», Lecture à l'Académie des Sciences morales et politiques, Paris, Institut de France, 1992, N4.

62 Ou, plus exceptionnellement, technique: «Analyse hiérarchique et coefficient de transitivité», in G. Michelat et J.-P. Thomas, Dimensions du nationalisme, Paris, Colin, 1966, 171-176 et «A model for the analysis of mobility tables», in: Blalock H., Aganbegian A., Borodkin F.M., Boudon R., Capecchi V. (éds.), Quantitative Sociology, New York, Academic Press, 1975, 511 527 /Moscou, Izdatel'stvo Mir, 1977. 
où j'avais été recruté à mon retour de Columbia, grâce en particulier à l'amicale bienveillance d'A. Touraine. Depuis le temps de ma double expérience allemande (j'avais passé ma deuxième année d'école normale à l'université de Fribourg en Brisgau) et américaine, j'avais en effet considéré - et je considère encore aujourd'hui - que l'Université est, en principe, le système organisationnel le plus propice à la création du savoir, celui dans lequel se cultivent le plus aisément les valeurs de la discussion et de l'échange entre les disciplines. La qualité impressionnante de certaines discussions scientifiques, comme celle qui a pendant des décennies interminablement soupesé L'éthique protestante de Weber, aurait été impensable sans l'existence d'un réseau d'universités et d'universitaires exigeants $^{63}$. Dans Le juste et vrai, j'ai insisté sur le fait que la plupart des théories sociologiques des normes se situent dans un cadre fixé notamment par Hume puis par le positivisme viennois, celui d'une coupure entre l'être et le devoir-être. N'est-il pas essentiel pour le sociologue de prendre conscience du fait que, dès qu'il analyse les phénomènes moraux, il pense à son insu dans ce cadre? Si on ne voit pas qu'il est implicitement traité par Pareto, Marx ou Freud comme une évidence, on ne comprend pas que ces auteurs développent une théorie irrationaliste des normes. De façon générale, Hume a fait le lit des explications irrationalistes des comportements et des croyances dans les sciences humaines. N'a-t-on pas là un exemple démontrant que la sociologie ne peut ignorer la philosophie, tant elle en est tributaire? De même, elle ne peut, pas plus que ne le peuvent l'économie ou la philosophie, ignorer les expériences de la psychologie cognitive ou de la psychologie sociale.

Mais l'Université ne facilite pas seulement les échanges entre disciplines. Elle est aussi un lieu naturel du brassage entre générations, sans lequel il n'est pas de vie scientifique. J'ai pour ma part souvent tiré davantage de profit des objections de mes étudiants que de celles de collègues prévenus ou trop polis. En tout cas, je ne crois pas pour ma part que j'aurais pu m'adonner à la recherche sans enseigner, et je n'éprouve jamais de joie professionnelle plus grande que lorsque, au cours de l'année de maîtrise ou de DEA, j'ai le sentiment de voir émerger un jeune chercheur ou lorsque, ayant dirigé une thèse, celle-ci me paraît constituer un apport à la connaissance suffisamment important pour mériter d'être lancée sur le marché.

Il me faut reconnaître toutefois que l'université française telle qu'elle est entretient un rapport un peu lointain avec celle dont il est permis de rêver.

Tourgéville/ Paris, août-septembre 1995

Université de Paris-Sorbonne (Paris-IV)

63 Boudon R., «L'Éthique protestante de Max Weber: le dossier de la discussion», Communication au colloque «Valeurs, comportements, développement, modernité: les facteurs culturels du sousdéveloppement et du développement», Paris, Institut de France, 15-16 septembre 1995, in Etudes sur les sociologues classiques, Paris, PUF, 1998. 